\title{
Verifying the Performance Characteristics of the (micro) Robotic Devices
}

\author{
J. Hricko, Š. Havlík, Yu. L. Karavaev
}

The paper is focused to design, simulation and modeling of the compact compliant structures widely used in construction of robotic devices. As the illustrative example it is proposed mechanism for reduction of motion, which enables to improve the accuracy of the positioning system. The physical model is fabricated by 3D printing technology. Its proposed performance characteristics are verified by measurement on the experimental test bed by using laser distance sensors and image sensing/processing technology.

Keywords: compact compliant mechanisms, 3D printing, modeling and simulation, HIL simulations

Received June 03, 2019

Accepted November 14, 2019

This work was supported by the Slovak Research and Development Agency under the contract No.: APVV-14-0076 - "MEMS structures based on load cell", and by the national scientific grant agency VEGA under project No.: 2/0155/19 — "Processing sensory data via Artificial Intelligence methods".

\author{
Jaroslav Hricko \\ hricko@savbb.sk \\ Stefan Havlík \\ havlik@savbb.sk \\ Institute of Informatics, Slovak Academy of Sciences \\ Banská Bystrica, Slovakia \\ Yury L. Karavaev \\ karavaev_yury@istu.ru \\ M. T. Kalashnikov Izhevsk State Technical University \\ ul. Studencheskaya 7, Izhevsk, 426069 Russia
}




\section{Introduction}

The miniaturization of the mechatronic devices lead to the utilization of the novel approaches to the devices design. In the case of (micro) mechatronic devices, it is clear that classic constructions based on the assembly from discrete parts cannot satisfy desired requirements. The single solutions are compact structures using micro-system technology or other precise production methods. Such structures can be used as compliant mechanisms in micropositioning devices, actuators for optoelectronics, micro-surgery, etc. A large group of MEMS devices represents micro-actuators and sensors (e.g. force/torque, pressure, speed, acceleration, flow rate...) [1].

In solving such mechanical structures specific approaches to design, kinematic, force and flexural analysis are used. Nevertheless, there is still a need to improve the quality of the mathematical description of micro-electro-mechanical systems (MEMS). These models, in general, are based on assumption that deflections and final performance characteristics of compliant mechanisms are linear within the range of validity of Hooke's law. But results from ordinary used linear models and real measured data differ up to $3-10 \%$ [2].

In general, the performance characteristics of compliant mechanisms are relatively linear, but this follows from small motions of the device.

The nonlinear behavior of the device proves itself when deployed in a real environment where, for example, the temperature is not constant, leading to a change in the dimensions of the flexible elements and a significant change in performance characteristics. As a suitable example of the mentioned behavior utilization of a motion reduction device can be studied when the high precision positioning with high payload is required. The common precise positioning device usually used a stepper motor with the positional encoder the no-backlash high ratio reduction gear and the ball screw [5], but the study [3] shows that the positioning errors from the ball screw are present. Consequently that the motion reduction compliant mechanism can be utilized as a device those potential positional errors can minimize (see Fig. 1). The solution to such a device leads to a complex optimization problem. The movement of such devices is usually in the range of $10 \%$ of maximum device dimensions, on the other side, for minimizing positioning error to e. g. 10\% (from the positioning error $30 \mu \mathrm{m}$ to $3 \mu \mathrm{m}$ ) it is required considerable input displacement what have a direct impact to the dimensions of the whole device. Other mentioned problem could be the device's robustness and its dynamical properties. For instance, the thickness of the flexure hinges, if is this thickness bigger the mechanisms is stiffer, but the

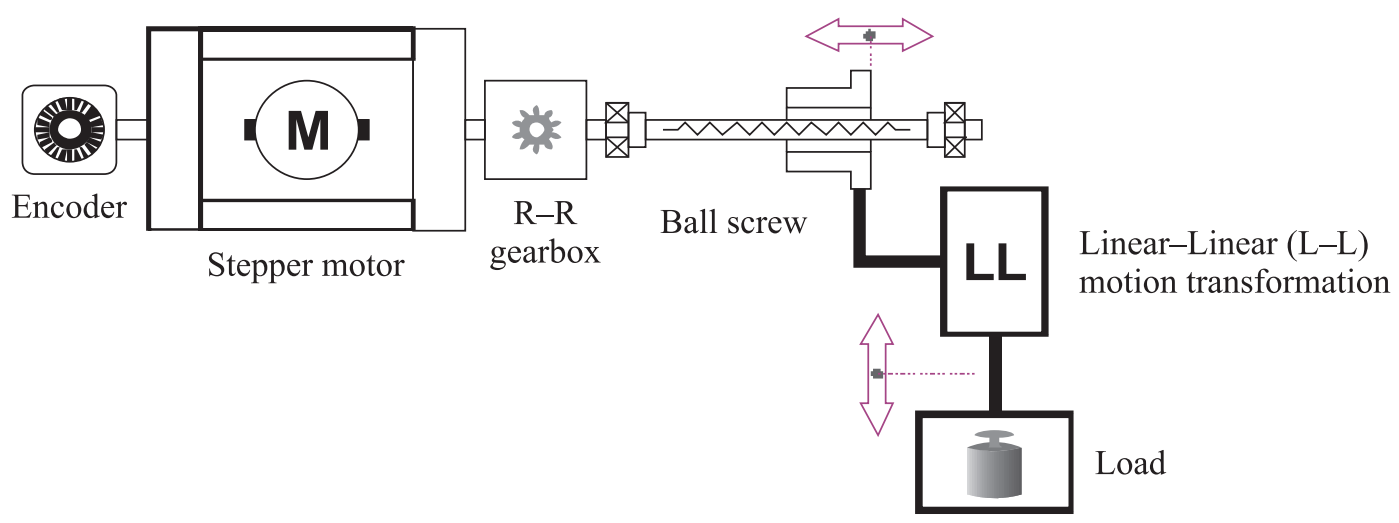

Fig. 1. The common servo mechanism with integrated motion reduction device [5]. 
arisen stress is higher, on the other side very thin hinges can lead to the unwanted oscillations, because mathematical expression of the compliant mechanism is mass-spring (damping) system.

\section{Design of the motion reduction device}

As a consequence of the minimal spatial possibilities, linear piezoelectric actuators are used in the area of the precise (micro) positioning. They can produce the desired displacement of several nanometers, but this displacement is in the range of $0.1 \%$ to $0.2 \%$ of their length [6]. To increase such displacement the mechanical amplifiers from one piece of material work on the principle elastic deformation are used. Between most know belongs differential mechanical amplifier, the amplifier working on the lever principle and triangular mechanical amplifier. For solving our problem (increase positioning precision of the common servo drive) some structures of mechanical amplifiers can work in to reverse mode. As shows our previous work [7], some new structures/configurations of the mechanical amplifiers cannot work in reverse mode, because the force at end of the mechanism is very small, and in the case of activities in reverse mode is this force smaller than stiffness of the whole mechanism, and therefore at the output of mechanism (input in standard mode) is zero displacement. As good examples can be referred research works [8] and/or some mechanisms designed by topology optimization approach. In the other side, lever and triangular mechanical amplifiers can work without any considerable restrictions in the reverse mode. From our analysis results, that Rhombus type amplifier enables to relatively large input displacement at the smallest external dimensions.

The desired performance of proposed mechanical motion reduction device is given as follows:

- The range of input displacement of the linear motion actuator to by $10 \mathrm{~mm}$;

- The range of output displacement should be $2 \mathrm{~mm}$, what corresponds with amplification ratio $R_{a m p}=0.2$;

- The load is given up to $100 \mathrm{~g} / 1 \mathrm{~kg}$.

As a manufacturing process will be used 3D printing technology, with which there are some limitations and boundaries, for instance, the minimal thickness of the flexure segments should be $0.5 \mathrm{~mm}$ optionally $0.8 \mathrm{~mm}$. As a material of the whole mechanism is considered Polylactic acid (PLA) with Young's modulus $E=2852 \mathrm{MPa}$, density $1237 \mathrm{~kg} / \mathrm{m}^{3}$, Poisson's ratio 0.36 and tensile stress at yield is $\sigma_{y}=38 \mathrm{MPa}$. Other alternative materials for building compliant mechanisms by 3D printing technology are Polypropylene (PP) or Polyamide (PA).

The acting load of the proposed physical mechanism was chosen to $100 \mathrm{~g}$, as a consequence of used linear actuator, that can produce the only $5 N$, however in analytical and numerical calculations will be taken into account $1 \mathrm{~kg}$ load, and the differences between proposed mechanisms will be mentioned below.

\subsection{Analytical approach}

In the literature, there are few approaches on how to calculate the amplification ratio $R_{a m p}$ (ratio between output displacement $o_{Y}$ and input displacement $i_{X}$ ). There are: the ideal model (geometric model) [9, 10], the elastic beam method (depends on the flexure elements Rhombus type - elastic arms and bridge type - flexure hinges) [11], matrix methods, Castigliano's second theorem [4] and method based on analysis of results from simulation software. In the following

RUSSIAN JOURNAL OF NONLINEAR DYNAMICS, 2020, 16(1), 161-172 
are described equations for calculation of the amplification ratio, force analysis, and stress calculation, with the assumption that one (output) side of the linear-linear motion transformation device is fixed to the frame.

The equation of amplification for ideal geometric model [10] is

$$
R_{a m p I}=\frac{o_{Y}}{L_{1} \sin \theta-L_{1} \sqrt{1-\left(\frac{1}{L_{1}}\left(L_{1} \cos \theta+\frac{i_{X}}{2}\right)\right)^{2}}}
$$

where $L_{1}$ is length of the flexible arm, $\theta$ is the angle between elastic arm and the direction of the input displacement. Unfortunately, (2.1) offer only basic and very rough results of the amplification radio, and could be useful for very small displacements (in range of nanometers). The equation based on elastic beam method for the Rhombus type of mechanical amplifier [11] is expressed as

$$
R_{a m p E}=\frac{K_{x F x} L_{1}^{2} \sin \theta \cos \theta}{12 K_{\theta z M z} \cos ^{2} \theta+K_{x F x} L_{1}^{2} \sin ^{2} \theta}
$$

where $K_{x F x}$ and $K_{\theta z M z}$ are elements of the stiffness matrix of corner-filleted flexure hinge (see [4], there are marked as $K_{1, x-F x}$ and $K_{1, \theta z-M z}$ ).

(a)

Corner-filleted flexure hinge ${ }_{\uparrow} F_{y}$



Rhombus mechanical amplifier

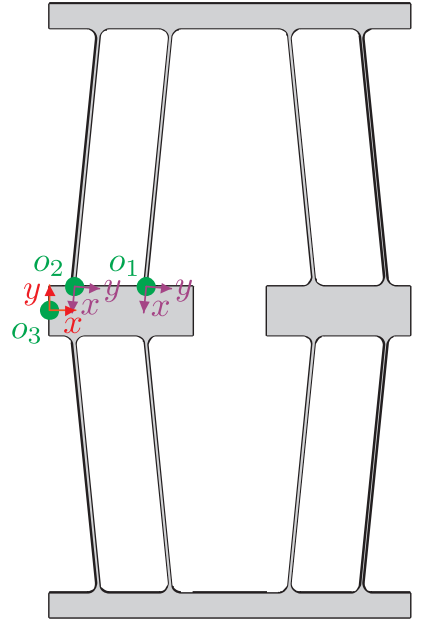

(b)

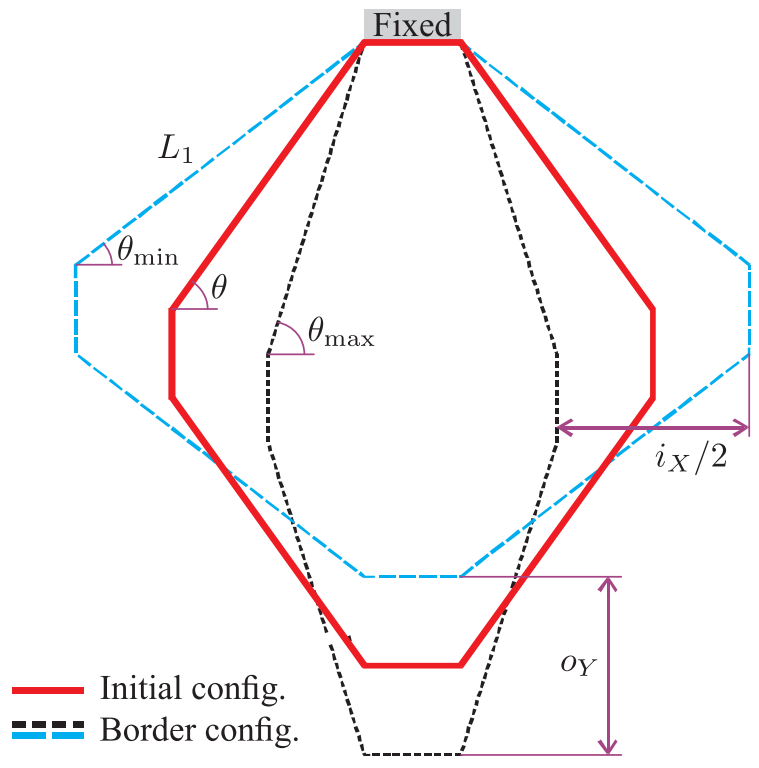

Fig. 2. a) Compliant body of the proposed Rhombus mechanical amplifier with local coordinate systems for stiffness model, b) sketch of the various positions of the motion reducer.

Forces analysis of such type device is based on the known amplification ratio and balance of forces. Considering the symmetrical of the compliant structure the analysis of the half mechanism can be evaluated and results can be applied to the whole device. The balance of forces can be evaluated by

$$
F_{\text {out }}=F_{\text {elastic }}-F_{\text {in }}
$$


where $F_{\text {out }}$ is force located on output of the mechanism equal to maximum load, $F_{\text {elastic }}$ is force required to create deformation (displacement, rotation) of flexure hinges and $F_{i n}$ is force of the actuator.

In the initial mechanism configuration (ideal model) is $F_{\text {elastic }}$ equal to zero and ac-cording to conservation of energy the dependence between input and output forces is equal

$$
\begin{aligned}
\frac{1}{2} F_{\text {in }} \Delta_{x}=\frac{1}{2} F_{\text {out }} \Delta_{y} & \Rightarrow \frac{1}{2} F_{\text {in }} \Delta_{x}=\frac{1}{2} F_{\text {out }} 2 \Delta_{x} R_{\text {amp } E}, \\
F_{\text {in }} & =2 R_{\text {amp } E} F_{\text {out }} .
\end{aligned}
$$

For the calculation of real forces model it is necessary to evaluate $F_{\text {elastic }}$. For this reason the stiffness model of whole structure should be performed. It was adapted matrix method described in $[12,13]$ and for the calculation of compliance/stiffness matrixes [4]. As was mentioned, such mechanisms works in the range of validity of the Hook's Law, and therefore, it is possible to write dependence between force/load $\mathbf{F}=\left[F_{x}, F_{y}, F_{z}, M_{x}, M_{y}, M_{z}\right]^{T}$, deflection $\mathbf{u}=\left[u_{x}, u_{y}, u_{z}, \theta_{x}, \theta_{y}, \theta_{z}\right]^{T}$ and compliance/stiffness $\mathbf{C}=\mathbf{K}^{-1}$, that express material mechanical properties, the shape of flexure and dimensions.

$$
\mathbf{u}=\mathbf{C F} \quad \rightarrow \quad \mathbf{F}=\mathbf{K u}
$$

The compliance matrix $\mathbf{C}$, is expressed as

$$
\mathbf{C}_{j}=\left(\begin{array}{cccccc}
C_{x F x} & 0 & 0 & 0 & 0 & 0 \\
0 & C_{y F y} & 0 & 0 & 0 & C_{y M z} \\
0 & 0 & C_{z F z} & 0 & C_{z M y} & 0 \\
0 & 0 & 0 & C_{\theta x M x} & 0 & 0 \\
0 & 0 & C_{z M y} & 0 & C_{\theta y M y} & 0 \\
0 & C_{y M z} & 0 & 0 & 0 & C_{\theta z M z}
\end{array}\right)
$$

Because the element of compliance matrix $C_{\theta x M x}$ was in the [4] neglected, the evaluation of this is (simplify version equal to beam flexure hinge [14])

$$
C_{\theta x M x}=\frac{L_{1}}{G a b^{3}\left(\frac{16}{3}-3.36 \frac{b}{a}\left(1-\frac{b^{4}}{12 a^{4}}\right)\right)}
$$

where $G$ is shear modulus, $a=w / 2(w-$ width of the flexure), $b=t / 2(t-$ thickness of flexure).

The compliance matrices of separate flexures are expressed to a specific endpoint (it is mean that matrix is related to the local coordinate system). For calculation of compliance/stiffness matrices of the whole structure, it is necessary to transform it into a global coordinate system. The transformation matrix expressed point located in the first coordinate system $\left(O_{2}\right)$ to the second coordinate system $\left(\mathrm{O}_{3}\right)$ is

$$
\mathbf{T}_{23}=\left[\begin{array}{cc}
\mathbf{R}_{23} & -\mathbf{R}_{23} \mathbf{P}_{23} \\
0 & \mathbf{R}_{23}
\end{array}\right]
$$


where $\mathbf{R}_{23}$ is rotation matrix between coordinate systems and $\mathbf{P}_{23}$ is position matrix of point $O_{3}$ expressed in reference coordinates $\mathrm{O}_{2}$ (see Fig. 2a). According to configuration of compliant structure the whole compliance/stiffness matrix can be calculated by relations where (2.10) is for serial and (2.11) for parallel arrangement of joints with respect to the acting force

$$
\begin{aligned}
\mathbf{C}_{23} & =\sum_{n} \mathbf{T}_{23}^{*} \mathbf{C}_{12}\left(\mathbf{T}_{23}^{*}\right)^{T}, \\
\mathbf{K}_{23} & =\sum_{n} \mathbf{T}_{23}^{*} \mathbf{K}_{12}\left(\mathbf{T}_{23}^{*}\right)^{T} .
\end{aligned}
$$

Transformation matrices between particular local frames to that the compliance matrices were calculated are as follows

$$
\begin{aligned}
\mathbf{T}_{j 01} & =\left(\begin{array}{cc}
\mathbf{R}_{z}(\pi+\theta) & 0 \\
0 & \mathbf{R}_{z}(\pi+\theta)
\end{array}\right) ; \\
\mathbf{T}_{23} & =\left(\begin{array}{cc}
\mathbf{R}_{z}(\pi-\theta) & -\mathbf{R}_{z}(\pi-\theta) \mathbf{P}_{x y z}\left(L_{x 23}, L_{y 23}, 0\right) \\
0 & \mathbf{R}_{z}(\pi-\theta)
\end{array}\right) ; \\
\mathbf{T}_{12} & =\left(\begin{array}{cc}
\mathbf{R}_{z}(0) & -\mathbf{R}_{z}(0) \mathbf{P}_{x y z}\left(L_{12} \sin \left(\frac{\pi}{2}-\theta\right),-L_{12} \cos \left(\frac{\pi}{2}-\theta\right), 0\right) \\
0 & \mathbf{R}_{z}(0)
\end{array}\right) .
\end{aligned}
$$

By substitution (2.12) to (2.10) and (2.11) it is possible to write the compliance matrix for point $O_{3}$ as follow

$$
\mathbf{C}_{23}=\mathbf{T}_{23}\left(\mathbf{T}_{12} \mathbf{T}_{j 01} \mathbf{C}_{j}^{-1} \mathbf{T}_{j 01}^{T} \mathbf{T}_{12}^{T}+\mathbf{T}_{j 01} \mathbf{C}_{j}^{-1} \mathbf{T}_{j 01}^{T}\right)^{-1} \mathbf{T}_{23}^{T} .
$$

To identify the size of $F_{\text {elastic }}$ only one element $\left(C_{23 x F x}=\mathbf{C}_{23}(1,1)\right)$ connected with acting force $F_{x}$ of matrix $\mathbf{C}_{23}$ is used. Then based on the symmetrical configuration of the proposed mechanism can be expressed force required for elastic deformation of the whole mechanism.

$$
F_{\text {elastic }}=2 \frac{1}{4 C_{23 x F x}} \Delta x \text {. }
$$

Except for calculations of the device movement and requirements to actuation force, it is the arisen stress important parameter of the design any compliant device. The arisen stress should be lower as the value of Yield point, for ensuring elastic deformation without permanent deformations. There are few approaches to calculate the maximal stress in the flexure hinge. According to our analyses, the combination results from the two approaches $[15,16]$ could provide values similar to FEM simulations for corner-filleted flexure hinge as follow

$$
\sigma_{\max }=\frac{6 K_{t} M_{z}}{t^{2} w}+\frac{K_{t a} F_{x}}{t w}
$$

where $K_{t}=\left(0.000594 e^{-4.99}+0.0000279 e^{0.153}\right)\left(\frac{L}{t}\right)^{2}+\left(-0.0346 e^{-4.86}-0.00394 e^{-0.292}\right) \frac{L}{t}+$ $+\left(0.547 e^{-4.34}+1.1 e^{-0.0293}\right)$ and $K_{t a}=-0.1729\left(\frac{r}{t}\right)^{3}+0.8539\left(\frac{r}{t}\right)^{2}-1.4265\left(\frac{r}{t}\right)+1.9613$. By substitution (2.6) to (2.15) it is possible to express the size of $M_{z}$ and $F_{x}$ as dependence of the corresponding element of the stiffness matrix and displacement or rotation produced by relevant force/torque. 


\subsection{FEM analysis}

The model in Comsol Multiphysics has been made as an optimization procedure of designed motion reducer. The topology of the mechanism proceeds from general Rhombus type amplifier, whereas a consequence of utilization in reverse mode the sides of input and output had been replaced. In common Rhombus mechanical amplifier, only one flexible arm is used, but the double flexure arms have used for increasing mechanism stiffness and robustness. As was mentioned above, the material properties and minimal thickness of flexure, input displacement and maximum force produced by linear actuator were input and boundary conditions that should be used. As a consequence of the varying amplification ratio $R_{a m p}$ (see Fig. 4) depended on input displacement the mechanism has been optimized with respect to achieving prescribed amplification ratio in specific state.

There ware studied cases with the precise displacement was in the range $i_{X} / 2$ from $-2.5 \mathrm{~mm}$ to $2.5 \mathrm{~mm}$ with various load from $0 N$ to $50 \mathrm{~N}$. Another case considers with force produced by linear actuator (verification, if on demanded actuator stroke is required force in the desired boundaries) and influence of load.

The results from the FEM approach shows that one flexible element of has length $L_{1}=40.8 \mathrm{~mm}$, thickness $t=0.8 \mathrm{~mm}$, width $w=10 \mathrm{~mm}$, and radius $r=2 \mathrm{~mm}$. The height of the whole mechanism is $120 \mathrm{~mm}$ and width $90 \mathrm{~mm}$ (the minimal available width can be $71 \mathrm{~mm}$, but for connection of the actuator was chosen bigger width).

In the Fig. 4 are shown output displacement $o_{Z}$ and amplification ratio $R_{a m p}$ for the selected studied cases. In this proposed mechanism the results (mainly output displacement) shown that the influence of the acting load has not to impact influence to output displacement. It is only registered small shift in the range of few-hundredth micrometers. The better figure of influence of the acting load shows dependences of amplification ratio. For the case of no load, the amplification ratio is relatively linear in the range from 0.16 to 0.24 . Rem.: depend on the

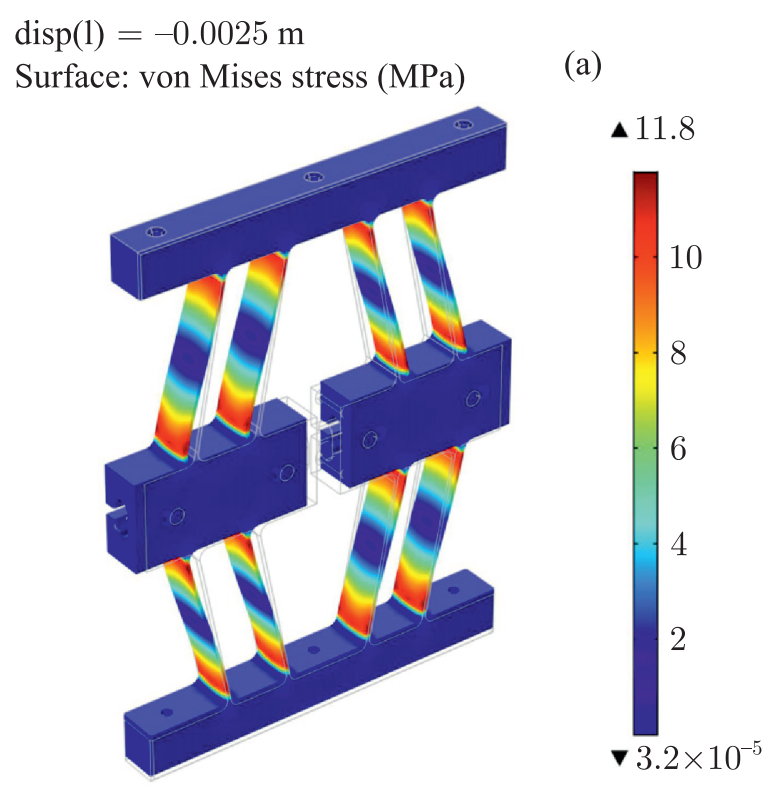

(b)

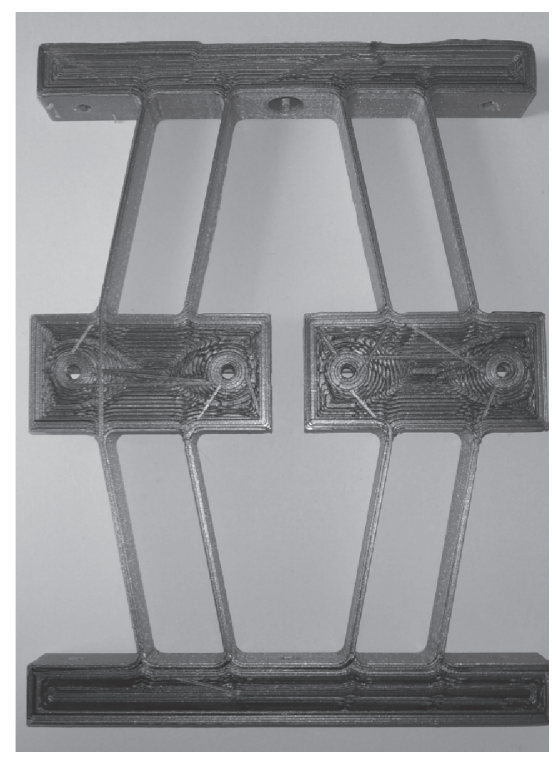

Fig. 3. a) Stress distribution in the proposed motion reduction device. b) 3D printed physical sample of motion reduction device. 
Dependence of output displacement $o_{Z}$ for various cases - FEM

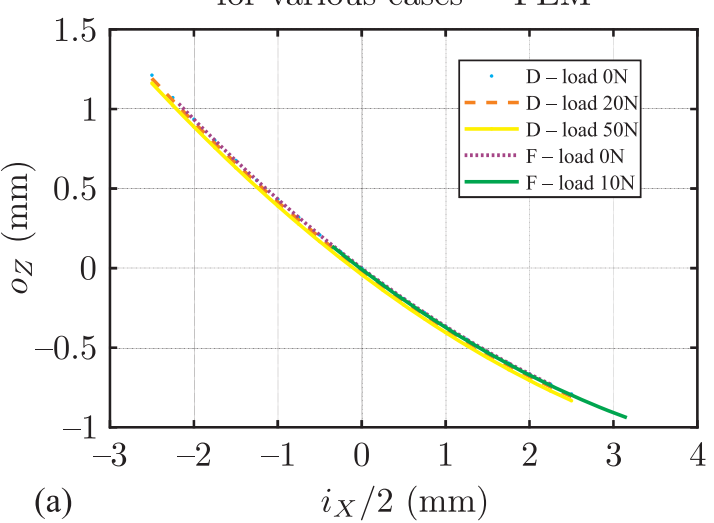

Amplification ratio $R_{a m p}$ for various cases - FEM

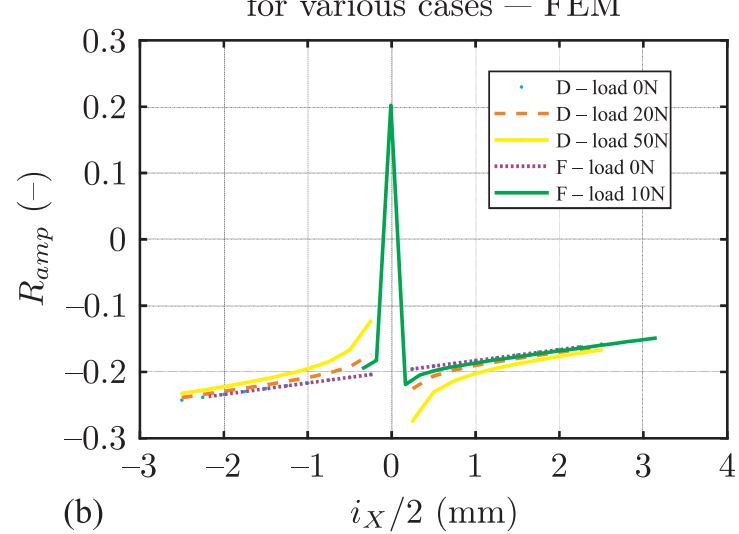

Fig. 4. Results from the FEM analysis a) dependence of output displacement for various cases of load and input (precise displacement $-D /$ force $-F$ ). b) Amplification ratio $R_{a m p}$ for various cases.

coordinate system in FEM software the displacement is reversing and therefore the graph shows a negative amplification ratio. For a better comparison of the results, the other data will be further inverted as well. The results show that in the case of the zero input displacement, the amplification ratio is increased into infinity. It is consequence of acting load and compliance of the flexure hinge. The simplify explanation is: when one flexure hinge is in vertical (see Fig. 2a) then acting load is $F_{x}$, and according to (2.6) is clear that this unwanted displacement is equal to size of acting load and compliance $C_{x F x}$ (see Eq. (2.7)).

\section{Experimental platform for tests of compliant structures}

Most adequate performance characteristics of a device can be obtained if a hybrid approach and corresponding models will be used. Considering the specific group of compliant robotic devices such an approach is really needed. This approach is strengthened by the fact that a compliant device includes mechanically flexible parts (joints/arms) as important building elements. Proposed experimental testbed enables to carry out functional tests and measurements on compliant devices as grippers, force/displacement amplifiers, etc. [17].

The whole testbed is built as a module system. The main components are linear actuator with stroke $10 \mathrm{~mm}$ that is controlled by own motor controller with position feedback with $30 \mu \mathrm{m}$ resolution. The actuator force is calculated by the measurement of the motor current. Additional force measurement is possible by force sensors (push, push-pull) with the range up $20 N$. The most important part of the experimental platform is a measurement of the small displacement of the tested devices. There ware chosen two approaches of small displacement measurement. The laser distance sensor enables to measure displacement with $1 \mu \mathrm{m}$ resolution and his sampling frequency $(2 \mathrm{kHz})$ enable to measure fast dynamical processes. Because the knowledge of flexure arms deformations can give a new type of feedback the camera with various types of the optical lens is used. In the case of the proposed mechanism, the output displacement is measured. All components of the experimental platform are in the industrial version. As the higher control system is used a computer with MATLAB software, which enables to realize HIL simulation, where the control algorithm can be running. The mutual communication between all components is providing through serial protocols like USB, RS485, etc. 

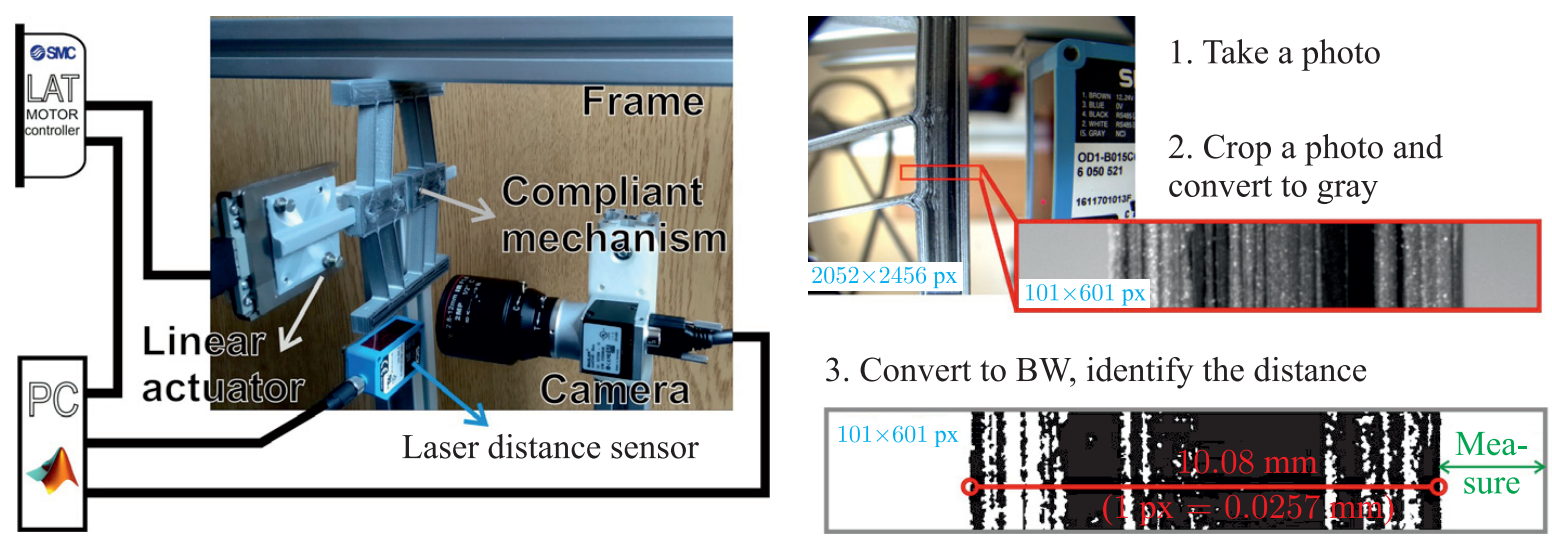

3. Convert to BW, identify the distance



Fig. 5. a) Scheme of the experimental platform for tests of CM. b) Image processing algorithm for measure output displacement of the proposed mechanism.

The algorithm of the measurement of the proposed motion reducer is programmed in the MATLAB environment. The initial step is establishing a connection with a linear actuator, laser displacement sensor, and a camera. Then the position of actuator had been set to the origin position. Next, it is run cycle when the position of the actuator is changed, then is taken a picture by the camera and read sets of measured values from the sensor. The output from the sensor is value in the micrometers. To the gained photography the image processing algorithm should be applied. The algorithm is shown in Fig. 5b. The photography from the camera is in the resolution $2052 \times 2456 \mathrm{px}$. From this image is a crop small area of interest with size $101 \times 601 \mathrm{px}$ (the area of the interest is located in the center of the taken photography for minimizing the influence of lens distortion). This image has been converted to the grayscale and black-and-white image. Next, it is measured the distance between the right corner and edge of mechanism. This value is converted from pixels to the millimeters. All measured data are stored for the next analysis.

\subsection{Comparison of results and discussion}

The measurement of characteristics has been made by the described algorithm. Unfortunately, the stiffness of the physical sample is higher as expected, what has a direct impact on possibilities of the linear actuator and his capability produce enough to satisfy the force. Consequently, only measurement of the mechanism without acting load and in the input displacement between $0.2 \mathrm{~mm}$ to $8.6 \mathrm{~mm}$ has been realized.

In Fig. $6 \mathrm{a}$ is shown dependences of the output displacement $o_{Z}$ for the case of moving mechanism without load. The characteristic shows results of the measurement on the 3D printed physical model (camera. laser) and the results from the FEM analysis and from the idealized geometric models (Eqs. (2.1) and (2.2)). From idealized amplification, ratios have been calculated output displacement. The results show that analytical and numerical approaches provide similar results with increasing difference between results when the input displacement is increased from the initial value. Considerable differences are by comparison of measured data and results from the models. The input displacement range of similar results from all observed approaches is only $i_{X} / 2= \pm 0.5 \mathrm{~mm}$.

As shows comparison graphs on Fig. 6, the behavior of the proposed mechanism is nonlinear and what is disagreeable the results from mathematical models and real device are diverse. 

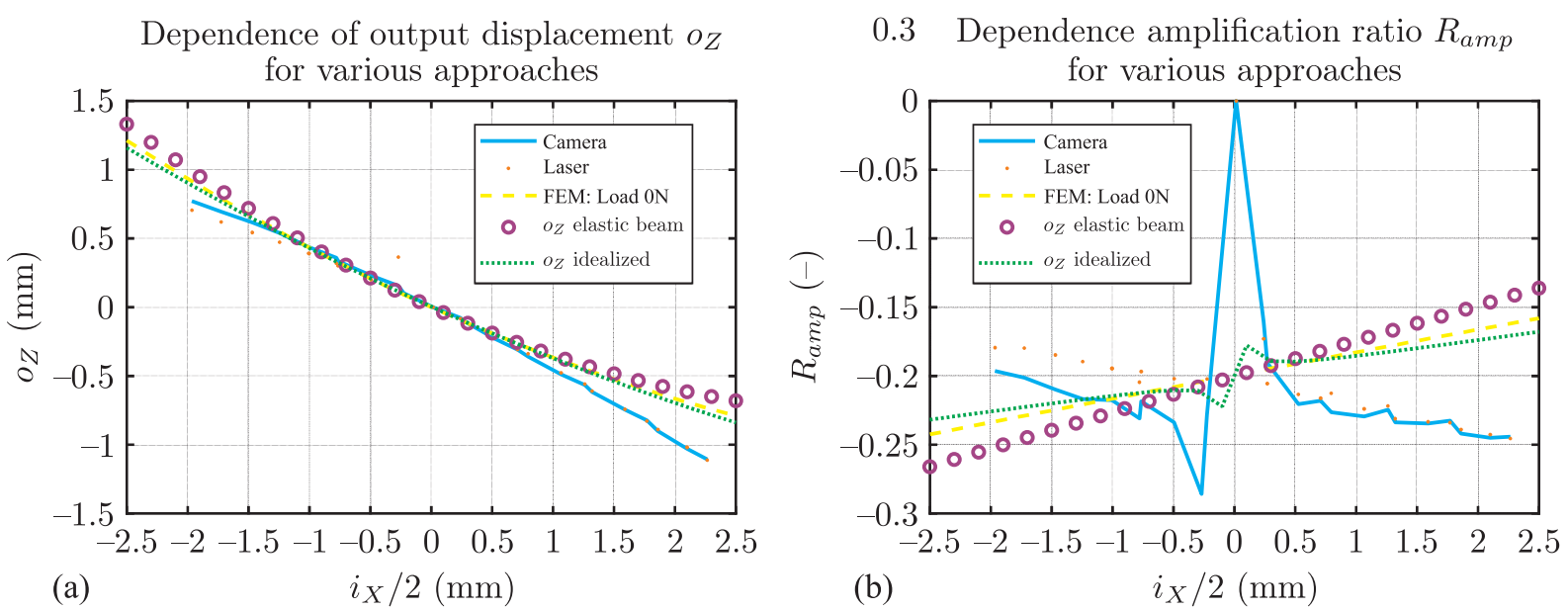

Fig. 6. a) Comparison of the output displacement of real device and mathematical models. b) Comparison of the $R_{a m p}$ of the proposed motion reducer for various approaches.

This has an impact on the design of the control system. In the ideal case, the control system can realize predictive control when the mathematical model is included. Unfortunately, this approach could not lead to acceptable results. Therefore position feedback is required. There are some possibilities like contactless optical methods (laser distance sensor, camera and image processing) unfortunately, these methods require additional space and good light conditions. Another solution is utilizing strain-gauge sensors [18], but they have some known issues. As an applicable approach, it seems using the new wireless principles to measure small displacements. One of it is described in the research works [19-22], where the electromagnetic principle is utilized. The principle of measurement is based on the detecting parameters changes in the electromagnetic field in which is placed the proposed mechanical device. In the device is integrated parallel resonance circuit, and in the case of its deformation (work) is changed the circuit resonance frequency that affects parameters of the electromagnetic field from the emitter. These changes are easy identified and converted to the value of arisen displacement with very high precision. The advantages of this approach are its wireless transfer of measured signals and the fact that measured data are transmitted by the frequency that is the most measurable quantity.

\section{Conclusion}

This paper deals with the problem of how to design precise robotic devices based on compliant mechanical structures/mechanisms. As obvious, no mathematical modes used for calculations in the design of such mechanisms can guarantee that desired parameters and performance will be satisfied. Any mathematical models provide only principal information about expected behavior and real characteristics differ from required. For this reason, it is always recommended to build the physical model and to verify its performance by measurements. On the example of the compliant motion reduction device is shown that errors due to cross-compliance effects and nonlinearities play an important role in cases of high accuracy devices. The design procedure of such devices with compliant mechanisms then consists of careful mathematical modeling and flexural analysis (analytical, numerical, FEM), simulation techniques, as well as verification of desired characteristics on physical models built on some scale by using suitable material and 
technology (3D print). In this example this procedure is discussed in more details and experimental equipment is described. It is documented that the presented approach enables to improve desired characteristics of a device, yet in the design phase.

As a suitable position feedback ware indicated a new electromagnetic approach [20, 22], where only parallel resonance circuit (capacitor and inductor) is situated on the compliant device. This minimizes additional space requirements. The importance of a strong control system with appropriate position feedback increases with increasing load, where the non-linear behavior of the motion reduction device increases disproportionately.

\section{References}

[1] Howell, L.L., Magleby, S.P., and Olsen, B. M., Handbook of Compliant Mechanisms, New York: Wiley, 2013.

[2] Linß, S., Schorr, P., and Zentner, L., General Design Equations for the Rotational Stiffness, Maximal Angular Deflection and Rotational Precision of Various Notch Flexure Hinges, Mech. Sci., 2017, vol. 8, no. 1, pp. 29-49.

[3] Zhang, Y., Pan, S., and Deng, J., Methods for Measuring and Compensating Ball Screw Error on Multi-Mode Industrial CT Scanning Platform, in Proc. of the 5th Internat. Conf. on Measurement, Instrumentation and Automation (Shenzhen, China, 2016).

[4] Lobontiu, N., Compliant Mechanisms: Design of Flexure Hinges, Boca Raton, Fla.: CRC, 2002.

[5] Havlík, Š., Hricko, J., Prada, E., and Jezný, J., Linear Motion Mechanisms for Fine Position Adjustment of Heavy Weight Platforms, in Proc. of the 28th Internat. Conf. on Robotics in Alpe-AdriaDanube Region (RAAD, Kaiserslautern, Germany, 2019), K. Berns, D. Görges (Eds.), Advances in Intelligent Systems and Computing, vol. 980, Cham: Springer, 2020, pp. 19-25.

[6] Dong, W., Chen, F., Gao, F., Yang, M., Sun, L., Du, Zh., Tang, J., and Zhang, D., Development and Analysis of a Bridge-Lever-Type Displacement Amplifier Based on Hybrid Flexure Hinges, Precis. Eng., 2018, vol. 54, pp. 171-181.

[7] Hricko, J. and Havlík, Š., Compliant Mechanisms for Motion/Force Amplifiers for Robotics, in Proc. of the 28th Internat. Conf. on Robotics in Alpe-Adria-Danube Region (RAAD, Kaiserslautern, Germany, 2019), K. Berns, D. Görges (Eds.), Advances in Intelligent Systems and Computing, vol. 980, Cham: Springer, 2020, pp. 26-33.

[8] Kota, S., Hetrick, J., Li, Z., and Saggere, L., Tailoring Unconventional Actuators Using Compliant Transmissions: Design Methods and Applications, IEEE/ASME Trans. Mechatronics, 1999, vol.4, no. 4, pp. 396-408.

[9] Chen, J., Zhang, C., Xu, M., Zi, Y., and Zhang, X., Rhombic Micro-Displacement Amplifier for Piezo-Electric Actuator and Its Linear and Hybrid Model, Mech. Syst. Signal Pr., 2015, vol. 50-51, pp. 580-593.

[10] Lobontiu, N. and Garcia, E., Analytical Model of Displacement Amplification and Stiffness Optimization for a Class of Flexure-Based Compliant Mechanisms, Comput. Struct., 2003, vol. 81, no. 32, pp. 2797-2810.

[11] Ling, M., Cao, J., Zeng, M., Lin, J., and Inman, D. J., Enhanced Mathematical Modeling of the Displacement Amplification Ratio for Piezoelectric Compliant Mechanisms, Smart Mater. Struct., 2016, vol. 25, no. 7, Art. ID 075022.

[12] Sciavicco, L. and Siciliano, B., Modeling and Control of Robot Manipulators, 2nd ed., Adv. Textb. Control Signal Process., London: Springer, 2001.

[13] Xu, Q. and Li, Y., Stiffness Modeling for an Orthogonal 3-PUU Compliant Parallel Micromanipulator, in Proc. of the IEEE Internat. Conf. on Mechatronics and Automation (Luoyang, Henan, China, 2006), pp.124-129.

[14] Young, W. C., Roark's Formulas for Stress and Strain, 6th ed., New York: McGraw Hill, 1989.

RUSSIAN JOURNAL OF NONLINEAR DYNAMICS, 2020, 16(1), 161-172 
[15] Chen, G., Liu, X., and Du, Y., Elliptical-Arc-Fillet Flexure Hinges: Toward a Generalized Model for Commonly Used Flexure Hinges, ASME J. Mech. Des., 2011, vol. 133, no. 8, 081002, 9 pp.

[16] Hale, L. C., Principles and Techniques for Designing Precision Machines, PhD Dissertation, Cambridge, Mass.: MIT, 1999.

[17] Hricko, J. and Havlík, Š., Design of Compact Compliant Devices: Mathematical Models vs. Experiments, Am. J. Mech. Eng., 2015, vol.3, no. 6, pp. 201-206.

[18] Hoffmann, K., An Introduction to Measurements Using Strain Gauges, Darmstadt: HBM, 1989.

[19] Hart'anský, R., Smieško, V., and Rafaj, M., Modifying and Accelerating the Method of Moments Calculation, Computing and Informatics, 2017, vol. 36, no. 3, pp. 664-682.

[20] Hart'anský, R. and Halgoš, J., The Problem of RF Radiator with Force Detector, in Proc. of the 11th Internat. Conf. on Measurement (Smolenice, Slovakia, May 2017), Bratislava: Slovak Acad. of Sci., 2017, pp. 139-142.

[21] Hart'anský, R., Analysis of Omni-Directivity Error of Electromagnetic Field Probe Using Isotropic Antenna, Meas. Sci. Rev., 2016, vol. 16, no.6, pp. 287-293.

[22] Hart'anský, R., Slížik, J., and Maršálka, L., Dipole near Field Analysis: A Closed Form Calculation in Cartesian Coordinates, J. Electr. Eng., 2013, vol. 64, no. 5, pp. 327-330. 